\title{
Painful Diabetic Peripheral Neuropathy Study of Chinese Outpatients (PDNSCOPE): A Multicentre Cross-Sectional Registry Study of Clinical Characteristics and Treatment in Mainland China
}

Yuanjin Zhang · Shaowei Zhang · Liya Pan • Baojun Wang • Yuanlin Sun · Lijun Gao · Ling Wang • Lijuan Cui • Qing Zhang · Heng Shang · Suqin Jin · Xing Qin · Deqin Geng $\cdot$ Xiaorong Yu $\cdot$ Lin Yang $\cdot \mathrm{Li} \mathrm{Li} \cdot \mathrm{Zuoxiao} \mathrm{Li} \cdot$ Chaoli Yan · Hongbin Sun · Tao Sun · Baoxin Du · Junying Cao · Fengyun Hu · Jianhua Ma · Shengnian Zhou • Fengli Zhao · Wei Li · Jianming Zheng Yanhui Yi $\cdot$ Jianguo Xu $\cdot$ Bo Hu $\cdot$ Baoying Sheng $\cdot$ Zhaohui Li Zhong Zhao · Ting Yang $\cdot \mathrm{Ni}$ Wang $\cdot$ Hongdong Zhao $\cdot$ Dunzhu Mima $\cdot$ Huaiqian Qu $\cdot$ Yi Wang $\cdot$ Fuxia Song · Xinyi $\mathrm{Li} \cdot \mathrm{Nan} \mathrm{Li} \cdot$ Dongsheng Fan

Received: May 3, 2021 / Accepted: June 10, 2021 / Published online: August 7, 2021

(C) The Author(s) 2021

\section{ABSTRACT}

Introduction: This aim of this study was to delineate current clinical scenarios of painful diabetic peripheral neuropathy (PDN) and associated anxiety and depression among

Supplementary Information The online version contains supplementary material available at https:// doi.org/10.1007/s40122-021-00281-w.

Y. Zhang · D. Fan $(\bowtie)$

Department of Neurology, Peking University Third Hospital, 49th North Garden Road, Haidian

District, Beijing, China

e-mail: dsfan2010@aliyun.com

S. Zhang

Department of Endocrinology, Shenyang Weikang

Hospital, Shenyang, China

L. Pan

Department of Neurology, The Fourth Affiliated

Hospital of Guangxi Medical University, Liuzhou, China

B. Wang

Department of Neurology, Baotou Central Hospital, Baotou, China

Y. Sun

Department of Neurology, Panjin Central Hospital, Panjin, China patients in Mainland China, and to report current therapy and clinical practices.

Methods: A total of 1547 participants were enrolled in the study between 14 June 2018 and 11 November 2019. Recruitment was conducted using a multilevel sampling method. Participants' demographics, medical histories, glucose parameters, Douleur Neuropathique 4 Questionnaire (DN4) scores, visual analogue scale (VAS) pain scores, Patient Health Questionnaire 9 (PHQ-9) scores, Generalised Anxiety Disorder

L. Gao

Department of Neurology, Affiliated Hospital of Chengde Medical University, Chengde, China

L. Wang

Department of Endocrinology, Fukuang General Hospital of Liaoning Health Industry Group, Fushun, China

L. Cui

Department of Endocrinology, Bengang General Hospital of Liaoning Health Industry Group, Benxi, China

Q. Zhang

Department of Neurology, General Hospital of Ningxia Medical University, Yinchuan, China

H. Shang

Department of Gastroenterology, Cangzhou People's Hospital, Cangzhou, China 
7 (GAD-7) scores and therapies were recorded.

Results: The male-to-female ratio was 1.09:1 (807:740), and the mean age at onset was $61.28 \pm 11.23$ years. The mean DN4 score $( \pm$ standard deviation) was $4.91 \pm 1.88$. The frequencies of DN4 sub-item phenotypes were: numbness, 81\%; tingling, 68.71\%; pins and needles, 62.90\%; burning, 53.59\%; hypoaesthesia to touch, 50.16\%; electronic shocks, 43.31\%; hypoaesthesia to pinprick, $37.94 \%$; brushing, 37.82\%; painful cold, 29.61\%; and itching, $25.86 \%$. Age, diabetic duration,

S. Jin

Department of Neurology, The Second Hospital of Shandong University, Jinan, China

X. Qin

Department of Neurology, The First Affiliated Hospital of Xi'an Jiaotong University, Xi'an, China

D. Geng

Department of Neurology, The Affiliated Hospital of Xuzhou Medical University, Xuzhou, China

X. Yu

Department of Neurology, Linfen People's Hospital, Linfen, China

L. Yang

Department of Neurology, The First Affiliated

Hospital of Dali University, Dali, China

L. Li

Department of Gastroenterology, The First People's Hospital of Taian, Tai'an, China

\section{Z. Li}

Department of Neurology, The Affiliated Hospital of Southwest Medical University, Luzhou, China

C. Yan

Department of Endocrinology, The Affiliated Hospital of Inner Mongolia Medical University, Hohhot, China

H. Sun

Department of Neurology, Sichuan Academy of Medical Sciences, Sichuan Provincial People's Hospital, Chengdu, China

T. Sun

Pain Department, Shandong Provincial Hospital, Jinan, China

B. Du

Department of Neurology, Guangdong Traditional Chinese Medicine Hospital, Guangzhou, China depression history, PHQ-9 score and GAD-7 score were identified as risk factors for VAS pain score. Peripheral artery disease (PAD) was a protective factor for VAS pain score. For all participants currently diagnosed with PDN and for those previously diagnosed PDN, fasting blood glucose (FBG) was a risk factor for VAS; there was no association between FBG and VAS pain score for PDN diagnosed within 3 months prior to recruitment. Utilisation rate of opium therapies among enrolled participants was $0.71 \%$, contradiction of first-line guideline recommendation for pain relief accounted for

J. Cao

Department of Neurology, Tianjin Gangkou

Hospital, Tianjin, China

F. Hu

Department of Neurology, Shanxi Provincial People's Hospital, Taiyuan, China

J. Ma

Department of Neurology, The First Affiliated Hospital of Xinjiang Medical University, Ürümqi, China

S. Zhou

Department of Neurology, Qilu Hospital of Shandong University, Jinan, China

F. Zhao

Department of Neurology, Yuncheng Central Hospital, Yuncheng, China

W. Li

Department of Neurology, Henan Provincial People's Hospital, Zhengzhou, China

J. Zheng

Department of Neurology, Mindong Hospital of Fujian Medical University, Ningde, China

Y. Yi

Department of Neurology, The Second People's Hospital of Hunan Province, Brain Hospital of Hunan Province, Changsha, China

J. Xu

Department of Neurology, Guiyang Sixth Hospital, Guiyang, China

B. $\mathrm{Hu}$

Department of Neurology, Union Hospital, Tongji Medical Collage of Huazhong University of Science and Technology, Wuhan, China

B. Sheng

Department of Neurology, The First Affiliated Hospital of Jiamusi University, Jiamusi, China 
9.43\% (33/350) and contradiction of secondline guideline recommendation for opium dosage form was $0.57 \%(2 / 350)$.

Conclusion: Moderate to severe neuropathic pain in PDN was identified in $73.11 \%$ of participants. Age, diabetic duration, depression history, PHQ-9 score, GAD-7 score and FBG were risk factors for VAS pain scores. PAD was protective factor. The majority of pain relief therapies prescribed were in accordance with guidelines.

Trial registration: ClinicalTrials.gov identifier, NCT03520608, retrospectively registered, 2018-05-11.

Keywords: Age; Anxiety; Depression; DN4; Newly diagnosed; Painful diabetic neuropathy; Peripheral artery disease; Visual analogue scale

Z. Li

Department of Neurology, People's Hospital of Xinxing Country, Yunfu, China

Z. Zhao

Department of Neurology, Suzhou Municipal

Hospital, Suzhou, China

T. Yang

Department of Neurology, Tianjin Xiqing Hospital, Tianjin, China

N. Wang

Department of Neurology, Central Hospital of

Wafangdian, Dalian, China

H. Zhao

Department of Neurology, Nanjing First Hospital, Nanjing, China

D. Mima

Department of Neurology, Tibet Autonomous

Region People's Hospital, Lhasa, China

H. Qu

Department of Neurology, Liaocheng People's

Hospital, Liaocheng, China

Y. Wang

Department of Neurology, Huashan Hospital Fudan

University, Shanghai, China

\section{Key Summary Points}

The frequencies of Douleur

Neuropathique 4 Questionnaire sub-item phenotypes were numbness, $81 \%$; tingling, 68.71\%; pins and needles, 62.90\%; burning, 53.59\%; hypoaesthesia to touch, $50.16 \%$; electronic shocks, 43.31\%; hypoaesthesia to pinprick, 37.94\%; brushing, 37.82\%; painful cold, 29.61\%; and itching, $25.86 \%$.

Age, diabetic duration, depression history, Patient Health Questionnaire 9 score and Generalised Anxiety Disorder 7 score were risk factors for pain intensity according to visual analogue (VAS) scale.

Peripheral artery disease was a protective factor for VAS pain score.

For all participants with diabetic peripheral neuropathy (PDN) and with previously diagnosed PDN, fasting blood glucose (FBG) was a risk factor for VAS pain score, and there was no association between FBG and VAS score for PDN diagnosed within 3 months prior to recruitment.

The majority of pain relief therapies were prescribed in accordance with guidelines.

F. Song

Department of Neurology, Yantai Yuhuangding Hospital, Yantai, China

X. Li

Department of Neurology, Shanxi Bethune Hospital, Taiyuan, China

N. Li $(\square)$

Research Center of Clinical Epidemiology, Peking

University Third Hospital, 49th North Garden Road, Haidian District, Beijing, China

e-mail: linan917@163.com 


\section{INTRODUCTION}

Diabetic peripheral neuropathy (DPN) is the most common complication of long-term diabetes and is a risk factor for foot ulceration and lower extremity amputation [1]. Patients with diabetes commonly suffer from diabetic polyneuropathy, usually affecting both large and small nerve fibres, and pure small fibre polyneuropathy; both conditions frequently cause neuropathic pain. When DPN is accompanied by painful symptoms, it is known as painful diabetic peripheral neuropathy (PDN). Some patients develop hyperalgesia, allodynia and simultaneous hyperaesthesia signs [2]. The reported prevalence of PDN ranges widely, as reported in studies with differing designs, diagnostic criteria and sampling methods. No recent study has evaluated the pattern of current medical practice at the national level in Mainland China. In the nationwide hospital-based study for PDN in Mainland China reported here, we assessed: (1) the prevalence of PDN clinical characteristics, including depression and anxiety symptoms; (2) the hypothesis that there are demographic and clinical risk factors associated with the visual analogue scale (VAS) pain severity score among participants with PDN; and (3) the hypothesis that there is a substantial gap between guideline recommendation compliance and current clinical practice treatments of pain relief.

\section{METHODS}

\section{Subjects and Study Design}

The Painful Diabetic Peripheral Neuropathy Study of Chinese Outpatients (PDN-SCOPE) is a multicentre cross-sectional registry study involving 51 hospitals distributed over 22 provinces, five autonomous regions and four municipalities in Mainland China. Participants were recruited from 14 June 2018 to 11 November 2019. Sample size estimation, participant selection and recruitment practices and procedures have been reported in an early publication [3]. Inclusion criteria were: (1) age $\geq 18$ years; (2) definite diagnosis of type 1 or 2 diabetes mellitus; (3) symptoms, signs and/ or electrophysiological evidence of DPN; (4) complaints of spontaneous pain (continuous or intermittent needle pricking, electric shock-like pain, burning pain, etc.) or induced pain (hyperalgesia and allodynia); (5) chronic pain for at least 3 months; and (6) signed informed consent. Exclusion criteria were: (1) no DPN, nociceptive pain or mixed pain (such as from the cervical/lumbar spine), degenerative disease, arthritis, nerve root compression, paraneoplastic syndrome, cerebral vascular disease, spinal cord disease and other peripheral neuropathies (immune, toxic, nutritional neuropathies, etc.); and (2) dementia, substance abuse or other conditions seriously impairing cognition and communication. Demographic, medical history and clinical characteristic variables were recorded and indexed. Pharmacotherapy medical coding was in accordance with the Medical Dictionary for Regulatory Activities and the Anatomical Therapeutic Chemical classification.

\section{Demographic, Clinical Characteristics and Patient-Reported Outcome Measures}

Marital status was defined as 'married' or 'unmarried' (with the latter category including widowed spouse and divorced individuals). Hypertension history and current antihypertension therapy were defined as per hypertension diagnosis. Hyperlipidaemia history and current lipid-lowering agent therapy were defined as per hyperlipidaemia diagnosis. Body mass index (BMI) was calculated as body weight $(\mathrm{kg}) /(\text { height }[\mathrm{m}])^{2}$. Participants completed patient-reported outcome measure questionnaires, including the Douleur Neuropathique 4 (DN4) Questionnaire [4], the VAS pain score, Patient Health Questionnaire-9 (PHQ-9), and Generalised Anxiety Disorder-7 (GAD-7). All scales were completed by participants during an outpatient visit. Where there were difficulties in reading or understanding, research investigators read the questions aloud and helped the participants complete the questionnaires. Details are described in the study protocol. 
Table 1 Differences in demographics, medical histories and clinical variables among participants with mild, moderate and severe pain

\begin{tabular}{|c|c|c|c|c|c|}
\hline Variables & Mild & Moderate & Severe & Statistical value & $P$ \\
\hline Age (years) & $59.21 \pm 11.21$ & $61.88 \pm 11.29$ & $62.34 \pm 10.87$ & $F 10.065$ & $<0.01^{*}$ \\
\hline Male participants & $55.53(231)$ & $52.15(376)$ & $48.54(199)$ & $\chi^{2} 4.054$ & 0.132 \\
\hline BMI $\left(\mathrm{kg} / \mathrm{m}^{2}\right)$ & $25.24 \pm 3.91$ & $24.97 \pm 10.20$ & $24.79 \pm 3.96$ & $F 0.37$ & 0.691 \\
\hline Marital status (married) & $94.23(392)$ & $94.17(679)$ & $91.22(374)$ & $\chi^{2} 4.334$ & 0.115 \\
\hline Smoking history (years) & $28.85(120)$ & $31.62(228)$ & $25.12(103)$ & $\chi^{2} 5.374$ & 0.068 \\
\hline Drinking history (years) & $22.36(93)$ & $17.34(125)$ & $14.88(61)$ & $\chi^{2} 8.256$ & $0.016^{*}$ \\
\hline Diabetic duration (months) & $129.18 \pm 88.93$ & $133.99 \pm 88.00$ & $155.64 \pm 95.51$ & F 10.496 & $<0.01^{*}$ \\
\hline DPN duration (months) & $39.61 \pm 42.63$ & $38.79 \pm 38.38$ & $55.30 \pm 51.99$ & F 20.993 & 0.044 \\
\hline PDN duration (months) & $28.09 \pm 36.03$ & $25.67 \pm 29.35$ & $35.27 \pm 40.56$ & F 10.305 & $0.001^{*}$ \\
\hline Hypertension & $56.01(233)$ & $55.89(403)$ & $57.80(237)$ & $\chi^{2} 5.891$ & 0.207 \\
\hline Hyperlipidemia & $48.08(200)$ & $54.92(396)$ & $55.37(227)$ & $\chi^{2} 6.018$ & $0.049^{*}$ \\
\hline Diabetic retinopathy & $22.84(95)$ & $23.58(170)$ & $21.46(88)$ & $\chi^{2} 0.664$ & 0.718 \\
\hline Diabetic nephropathy & $20.19(84)$ & $16.50(119)$ & $17.32(71)$ & $\chi^{2} 2.521$ & 0.284 \\
\hline Chronic heart disease & $25(104)$ & $27.18(196)$ & $30.49(125)$ & $\chi^{2} 3.177$ & 0.204 \\
\hline Stroke & $18.51(77)$ & $17.06(123)$ & $22.20(91)$ & $\chi^{2} 4.547$ & 0.103 \\
\hline Peripheral vascular disease & $18.51(77)$ & $26.91(194)$ & $26.91(194)$ & $\chi^{2} 87.887$ & $<0.01^{*}$ \\
\hline Depression disorder & $2.16(9)$ & $4.85(35)$ & $5.85(24)$ & $\chi^{2} 8.262$ & $0.016^{*}$ \\
\hline Anxiety disorder & $4.57(19)$ & $4.44(32)$ & $4.15(17)$ & $\chi^{2} 0.093$ & 0.955 \\
\hline Fasting blood glucose $(\mathrm{mmol} / \mathrm{L})$ & $8.61 \pm 3.10$ & $9.14 \pm 3.52$ & $9.43 \pm 3.80$ & $F 5.906$ & $0.003^{*}$ \\
\hline Postprandial blood glucose $(\mathrm{mmol} / \mathrm{L})$ & $13.17 \pm 4.52$ & $13.09 \pm 4.50$ & $13.69 \pm 5.22$ & $F 2.248$ & 0.106 \\
\hline Glycosylated hemoglobin (\%) & $8.77 \pm 2.23$ & $8.58 \pm 2.16$ & $8.71 \pm 2.06$ & $F 1.163$ & 0.313 \\
\hline DN4 score & $3.61 \pm 1.41$ & $4.90 \pm 1.59$ & $6.24 \pm 1.84$ & F 272.102 & $<0.01^{*}$ \\
\hline PHQ-9 score & $1,3,5$ & $3,5,7$ & $4,6,10$ & Z-score 150.41 & $<0.01^{*}$ \\
\hline GAD-7 score & $1,2,4$ & $2,3,5$ & $2,5,7$ & Z-score 118.38 & $<0.01^{*}$ \\
\hline
\end{tabular}

$D N 4$ Douleur Neuropathique 4 Questionnaire, $D P N$ diabetic peripheral neuropathy, GAD-7 Generalised Anxiety Disorder 7, $P D N$ painful diabetic peripheral neuropathy, $P H Q-9$ Patient Health Questionnaire 9

${ }^{*}$ Drinking history, DPN duration, Hyperlipidemia, Depression disorder should be deleted since there is no statistical difference as definition $P<0.015$ 
Table 2 Differences in duration of newly diagnosed PDN, previous diagnosed PDN between the mild, moderate and severe pain groups

\begin{tabular}{llllll}
\hline Variables & $\begin{array}{l}\text { Mild } \\
(\mathbf{\%}, \mathbf{4 1 6})\end{array}$ & $\begin{array}{l}\text { Moderate } \\
(\mathbf{\%} \text { 721) }\end{array}$ & $\begin{array}{l}\text { Severe } \\
(\mathbf{\%} \text { 410) }\end{array}$ & $\begin{array}{l}\text { Statistical } \\
\text { value }\end{array}$ & $\boldsymbol{P}$ \\
\hline $\begin{array}{l}\text { New diagnosed before recruit within } \\
\quad 31.49(131)\end{array}$ & $37.17(268)$ & $31.46(129)$ & & \\
$\quad$ months & & & & & \\
Diabetic duration (months) & $81.83 \pm 82.43$ & $96.45 \pm 89.15$ & $107.40 \pm 99.37$ & $\mathrm{~F}=2.075$ & 0.127 \\
DPN duration (months) & $4.98 \pm 3.14$ & $5.65 \pm 3.13$ & $5.35 \pm 2.94$ & $\mathrm{~F}=1.659$ & 0.192 \\
PDN duration (months) & $0.19 \pm 0.47$ & $0.15 \pm 0.44$ & $0.23 \pm 0.51$ & $\mathrm{~F}=0.911$ & 0.403 \\
Previous diagnosed PDN & $68.51(285)$ & $62.83(453)$ & $68.54(281)$ & & \\
Diabetic duration (months) & $114.92 \pm 97.66$ & $121.74 \pm 90.75$ & $148.71 \pm 101.04$ & $\mathrm{~F}=11.214$ & $<0.01^{*}$ \\
DPN duration (months) & $5.05 \pm 3.34$ & $5.46 \pm 3.36$ & $5.28 \pm 3.14$ & $\mathrm{~F}=1.445$ & $<0.236$ \\
PDN duration (months) & $25.97 \pm 36.52$ & $23.84 \pm 28.71$ & $34.95 \pm 42.55$ & $\mathrm{~F}=9.942$ & $<0.01^{*}$ \\
\hline
\end{tabular}

${ }^{*}$ Statistically significant difference at $P<0.01$

\section{Determination of Contradiction with Guideline Standards}

The study describes actual PDN outpatient treatment and inappropriate drug use, with the latter defined as 'contradiction of existing guidelines'. The standards used are:

(1) Consensus for pharmacological treatment management of PDN by the Toronto Consensus Panel on Diabetic Neuropathy [5] was used to determine whether treatments were contradictions of recommended guidelines, or not. The recommended treatments are: physiological glucose control (glycosylated haemoglobin [HbA1c] 6-7\%) and management of cardiovascular risk factors. Pharmacological agents with proven efficacy for PDN include tricyclic antidepressants (TCA), selective serotonin and noradrenaline reuptake inhibitors (duloxetine and venlafaxine), anticonvulsants (gabapentin, pregabalin, tegretol and topiramate), opioids (tramadol and oxycodone), membrane stabilisers, the antioxidant alpha-lipoic acid and topical agents, including capsaicin.
(2) The evidence-based guidelines for treatment from the American Academy of Neurology (AAN) [6] recommend pregabalin as a level A treatment and other therapies (gabapentin, sodium valproate, venlafaxine, duloxetine, amitriptyline, dextromethorphan, morphine sulfate, tramadol, oxycodone, capsaicin, isosorbide dinitrate spray, electrical stimulation and percutaneous nerve stimulation) as level $\mathrm{B}$ treatments. Because there has been no update of treatment guidelines for PDN in China since 2013, we justifiably also used these guidelines as standards in this research.

(3) The American Association of Clinical Endocrinology (AACE) diabetes mellitus guideline algorithm for treatment of neuropathic pain [7], after exclusion of nondiabetic aetiologies, includes diuretics, splinting, lidocaine patch, surgical release for peripheral nerve compression, gabapentin, pregabalin, TCA, tramadol, oxcarbazepine, opioids and topicals. In this research, we defined pain relief (gabapentin, pregabalin, duloxetine, venlafaxine, tegretol and topiramate) and opiates, such as the synthetic opioid tramadol, morphine and controlled-release oxycodone, 


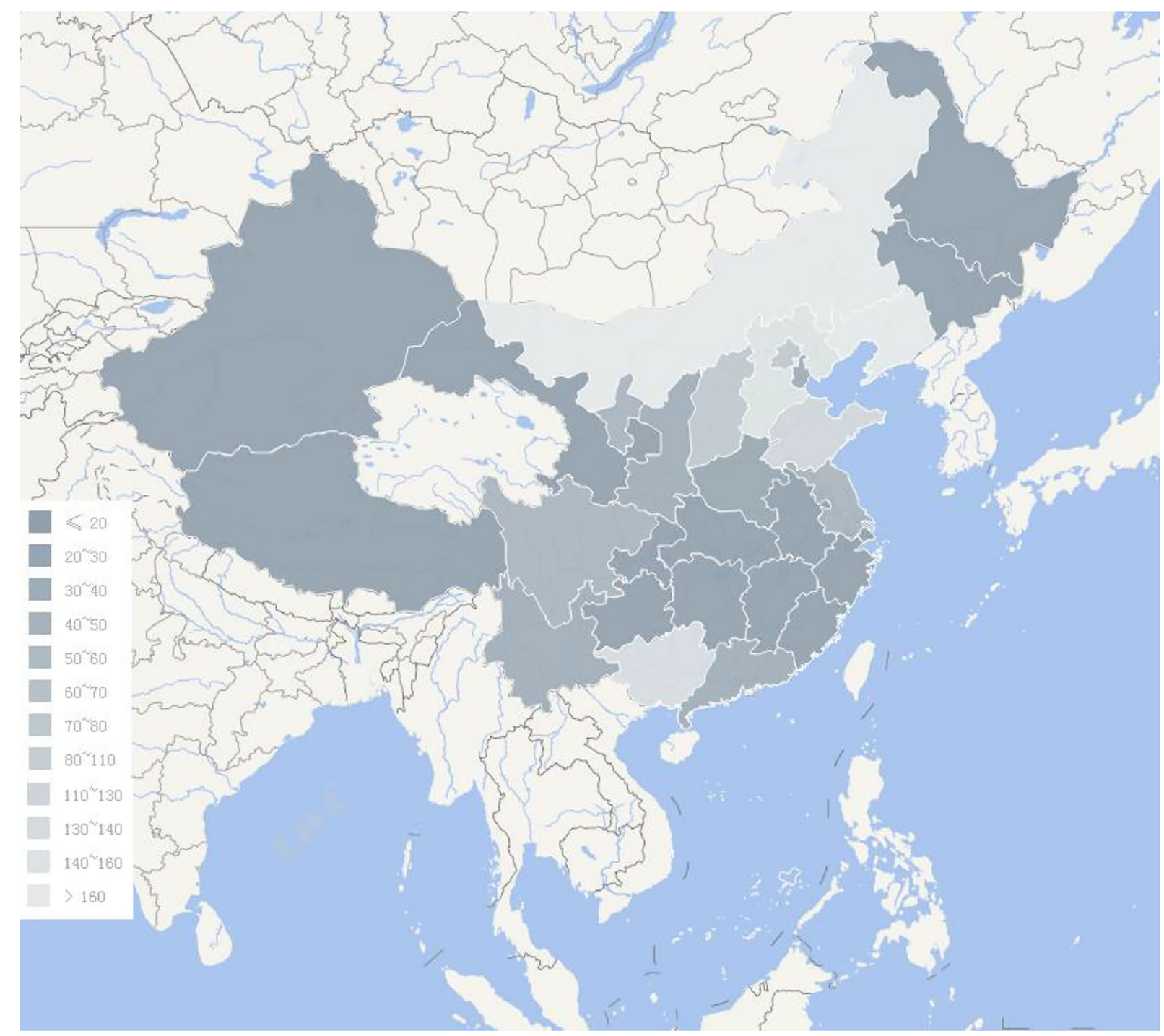

Fig. 1 The sites distribution of participants' enrollment among provinces, autonomous regions and municipalities

as guideline-recommended pain relief therapy. Oxcarbazepine, which is not recommended in the AAN guidelines but recommended by the AACE), was also defined as contradiction of guideline recommendation.

\section{Ethical Considerations}

All participants volunteered to participate in the SCOPE study and gave written, informed consent. Master ethical approval was obtained from the Peking University Third Hospital Medical Science Research Ethics Committee (Record Number: IRB00006761-M2018029, approval number: 2018-182-01). The study was performed in accordance with the Helsinki Declaration of 1964 and its later amendments. The ClinicalTrials.gov registration number is NCT03520608 (2018-05-11). Participating hospitals also acquired local site ethical approval. Ethic committees of several participating centres adopted a registry system without a reference number. Several ethics committees accepted the common practice that retrospective research did not require ethics approval. 


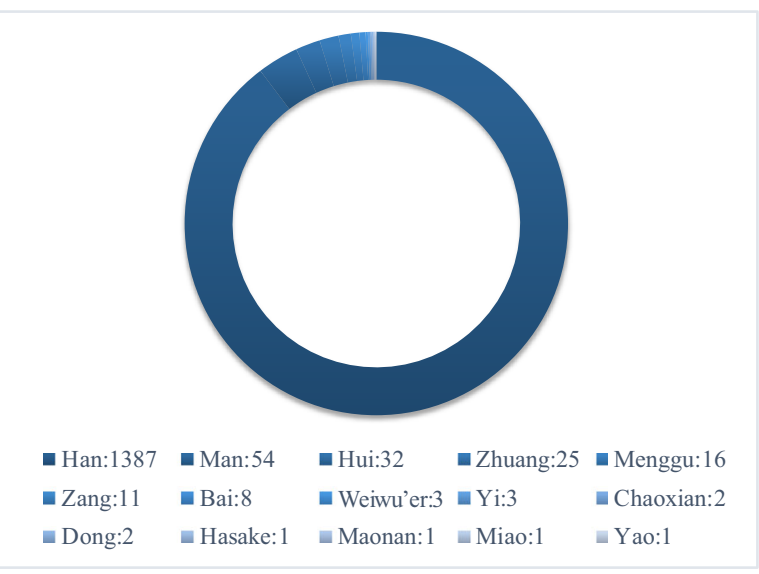

Fig. 2 The distribution of participants' nationalities

One survey participant was not approved for continuing investigation, and the data on this patient were not included in the statistical analysis.

\section{Establishment of Model}

The VAS score intervals $0-3,4-6$ and $7-10$ were defined as mild, moderate and severe pain, respectively. The factors entered in the generalised linear model (GLM) analysis were selected by single-factor analysis, with statistical significance set at $P<0.15$ at the first step. The dependent variable was VAS pain severity score. The fixed factors were gender, marital status, smoking status, alcohol status and comorbidities. The random factors were diabetic duration, DPN duration, PDN duration, DN4 score, PHQ-9 score, GAD-7 score and glucose values.

\section{Statistical Analysis}

Following the exclusion of 'frozen data' cases (four participants without actual data and one participant not approved for continuing investigation), 1547 cases were analysed. Age, BMI, fasting blood glucose (FBG), postprandial blood glucose (PBG), HbA1c and other continuous data were expressed as the mean \pm standard deviation (SD). On the basis of the normality test, diabetic duration, DPN duration, PDN duration, DN4 score, VAS score, PHQ-9 score and GAD-7 score were expressed as the mean \pm $\mathrm{SD}$, or as the median and $25 \mathrm{th}-75$ th percentile ranges, as appropriate. For continuous data that were normally distributed, parametric tests

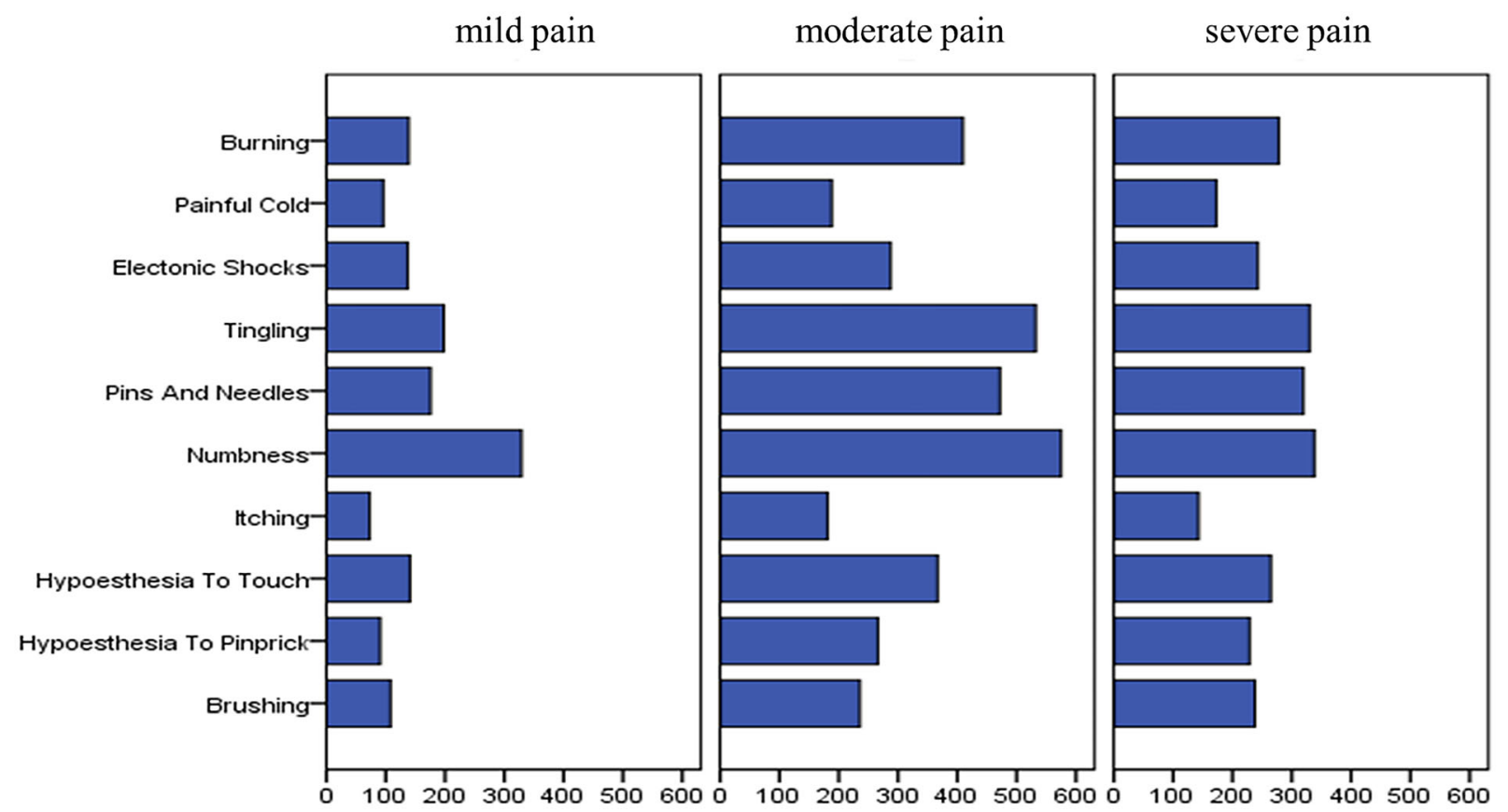

Fig. 3 The distribution of DN4 each item score among mild, moderate and severe pain severity according to VAS 

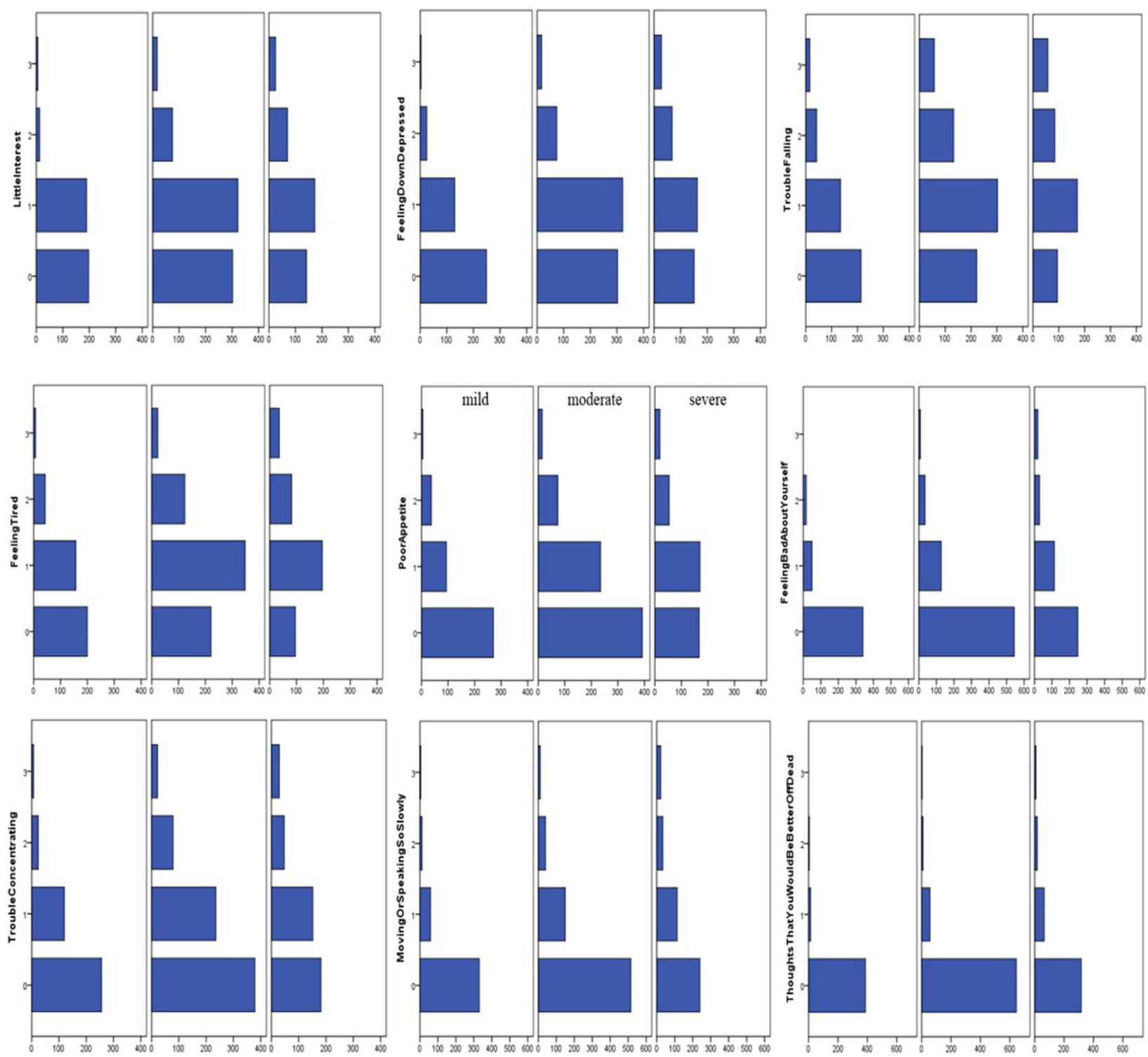

Fig. 4 The distribution of PHQ-9 and GAD-7 each item score among mild, moderate and severe pain severity according to VAS

(one-way analysis of variance) were used to compare mild, moderate and severe pain subgroup(s). For data that were not normally distributed, a non-parametric test (Kruskal-Wallis by rank) was used. Ethnicity was shown by a percentage graph. Gender, marital status, type of diabetes (type 1 or 2), comorbidities and other categorical data were expressed as the frequency and percentage. Categorical variables were calculated and compared using Chisquared and Fisher's exact tests. The distributions of pain, anxiety and depression severity were recorded using DN4, VAS, PHQ-9, and GAD-7 scores, and sub-item scores were described using a column diagram. GLM analysis was 

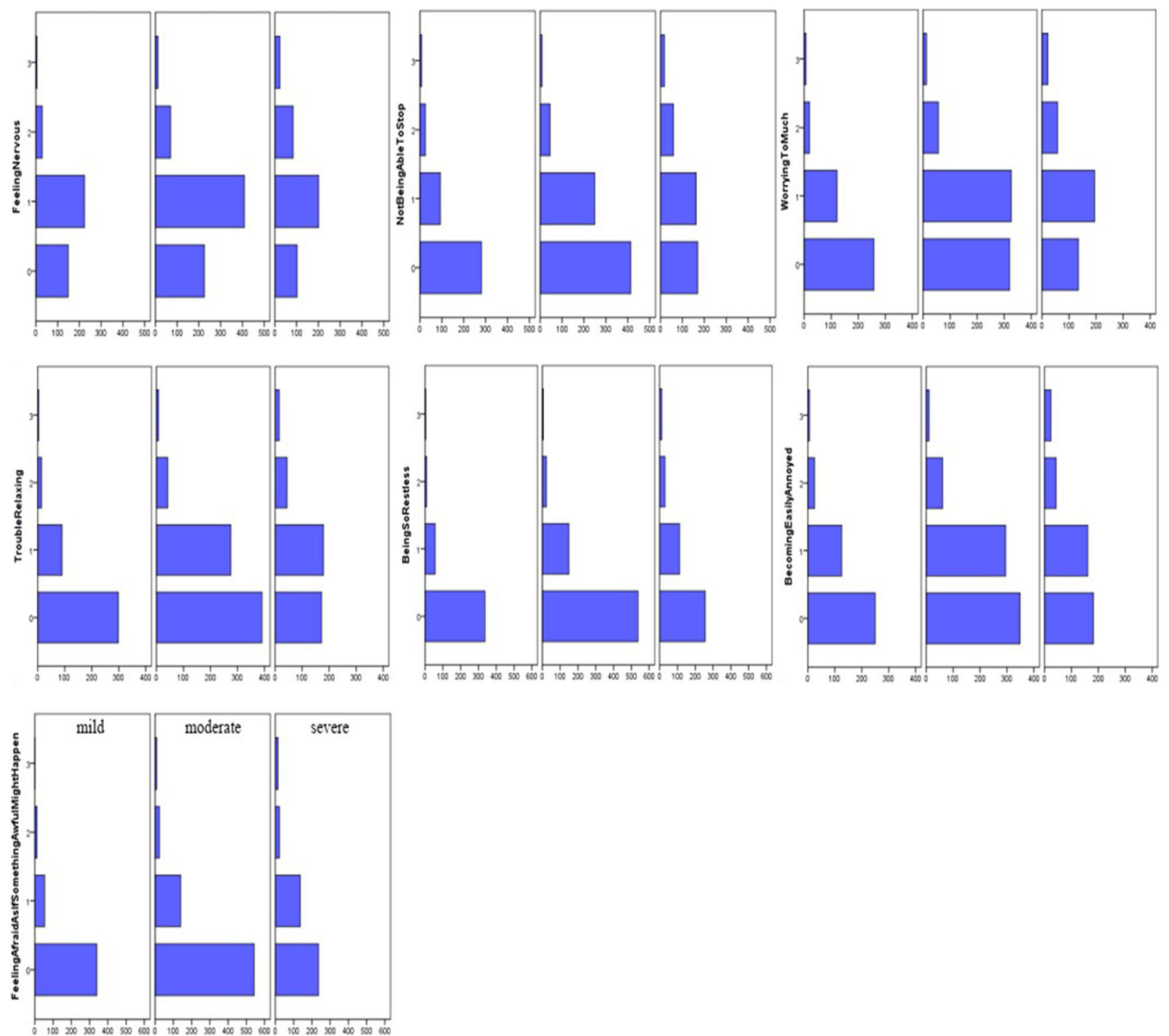

Fig. 4 continued

performed in order to examine the associations of risk factors and VAS pain severity.

The statistical package IBM ${ }^{\circledR}$ SPSS 22.0 (IBM, Armonk, NY, USA) for Windows was used for the analysis. Values of $P<0.05$ were considered to indicate statistical significance.

\section{RESULTS}

\section{Demographic and Clinical Characteristics of Participants}

A total of 1547 participants diagnosed with PDN were identified and recruited to the study. The distribution of these patients across provinces is shown in Fig. 1. Of these 1547 participants, 23 had type 1 diabetes and 1524 had type 2 diabetes. The mean age ( \pm SD) was 
$61.28 \pm 11.23(15-97)$ years. The proportion of men and women was $52.17 \%(807 / 1547)$ and $47.83 \%$ (740/1547), respectively. Mean BMI was $24.99 \pm 7.51 \mathrm{~kg} / \mathrm{m}^{2}$. The frequency of tobacco consumers and ex-smokers was $29.15 \%$ (451/ $1547)$ and $33.70 \%(152 / 451)$, respectively, and that of alcohol drinkers and abstainers was $18.03 \%(279 / 1547)$ and $32.97 \%$ (92/279), respectively. Married, divorced, unmarried and widowed participants accounted for $93.41 \%$ (1445/1547), 1.36\% (21/1547), 2.20\% (34/1547) and $3.04 \%(47 / 1547)$ of the study population, respectively. The distribution of nationalities is shown in Fig. 2. The diabetes duration was $138.43 \pm 90.85$ months; DPN duration was $43.39 \pm 44.05$ months; and PDN duration was $28.86 \pm 34.65$ months. Mean FBG, PBG and HbA1C values were $9.07 \pm 3.51 \mathrm{mmol} / \mathrm{L}$, $13.27 \pm 4.71 \mathrm{mmol} / \mathrm{L}$ and $8.67 \% \pm 2.16 \%$, respectively. The mean $\mathrm{DN} 4$ score was $4.91 \pm 1.88$. The frequency of DN4 sub-item phenotypes was: numbness, $81 \%$; tingling, $68.71 \%$; pins and needles, 62.90\%; burning, $53.59 \%$; hypoaesthesia to touch, $50.16 \%$; electronic shocks, $43.31 \%$; hypoaesthesia to pinprick, 37.94\%; brushing, 37.82\%; painful cold, $29.61 \%$; and itching, $25.86 \%$. The distribution of participants' somatosensory phenotype according to the DN4 score is shown in Fig. 3. The mean VAS pain score was $4.98 \pm 2.16$. The main comorbidities were hypertension $(56.43 \%$; 873/1547), hyperlipidaemia (53.20\%; 823/1547, peripheral artery disease $(30.12 \% ; 466 / 1547)$, cardiovascular disease $(27.47 \%$; 425/1547), diabetic retinopathy $(22.75 \% ; 352 / 1547)$, stroke (18.81\%; 291/1547), diabetic nephropathy $(17.71 \%$; $274 / 1547)$, depression disorder $(4.4 \%$; $68 / 1547)$ and anxiety disorder (4.4\%; 68/1547).

\section{Pain Severity Analysis and Association of PHQ-9 Scores and GAD-7 Scores}

The IQR of the PHQ-9 score was 2, 5 and 7.75 (range 0-25). The IQR of GAD-7 was 1, 3 and 6 (range 0-21). The distribution of each sub-item score for the PHQ-9 and GAD-7 is shown in Fig. 4, according to mild, moderate and severe pain intensity. PHQ-9 and GAD-7 scores were higher in participants reporting severe and moderate pain than in those reporting mild pain. The differences in the IQR for PHQ-9 and GAD-7 scores were statistically significant among mild (PHQ-9: 1, 3, 5; GAD-7: 1, 2, 4), moderate (PHQ-9: 3, 5, 7; GAD-7: 2, 3, 5) and severe (PHQ-9: 4, 6, 10; GAD-7: 2, 5, 7) pain groups $(P<0.01)$. Also, the differences in frequency of previous depression history were statistically significant among mild $(2.16 \%$, $9 / 416)$, moderate $(4.85 \%, 35 / 721)$ and severe $(5.85 \%, 24 / 410)$ pain groups $(P=0.016)$. The proportion of previous depression disorder was higher in those participants reporting moderate and severe pain groups than in those reporting mild pain. However, there was no statistically significant difference in previous anxiety history among mild $(4.57 \%, 19 / 416)$, moderate $(4.44 \%, 32 / 721)$ and severe $(4.15 \%, 17 / 410)$ pain groups $(P=0.955)$. These results are shown in Table 1.

\section{Differences in Risk Factors Among VAS Pain Severity Score}

The differences in VAS pain severity scores between the mild, moderate and severe pain groups are shown in Table 1. Age, diabetic duration, DPN duration, FBG, DN4 score, PHQ9 score and GAD-7 score in the severe, moderate pain group were higher than mild pain group with statistically significant differences. Previous depression disorder in the moderate and severe pain group was higher than that in the mild pain group with statistically significant differences. The frequency of peripheral vascular disease (PAD) was significantly higher in the severe and moderate pain groups than in the mild pain group.

According to the pre-defined significance level of $P<0.15$, the variables of age, gender, marital status, smoking history, drinking history, diabetic duration, DPN duration, PDN duration, hyperlipidaemia, stroke, PAD, previous depression disorder, FBG, PBG, DN4 score, PHQ-9 score and GAD-7 score among the mild, moderate and severe pain groups were included as independent variables in the GLM analysis. 


\section{Difference in Duration Between Newly Diagnosed PDN and Previously Diagnosed PDN}

In accordance to the inclusion and exclusion criteria, newly diagnosed PDN was defined as disease newly diagnosed within 3 months prior to recruitment to the study. We found no statistically significant difference in duration of diabetes, DPN and PDN among the mild, moderate and severe pain groups of new diagnosed PDN. There was statistical significance of diabetic and PDN duration among mild, moderate and severe pain groups of previous diagnosed PDN. The differences are shown in Table 2. The diabetic and PDN duration of previously diagnosed PDN in the severe pain group were more statistically significant than those in the moderate and in the mild pain groups.

\section{GLM Analysis for Risk Factors and VAS Pain Severity Score}

The GLM analysis was performed to determine whether there was an association between the demographics and clinical variables and VAS pain score among patients with PDN (see Appendix). The age, depression history, diabetic duration, PHQ-9 score and GAD-7 score were risk factors that were postively associated with VAS severity pain score. PAD was a predictive factor for VAS pain score in patients with DPN. FBG was positively correlated with VAS score for all cases of PDN and previously diagnosed PDN, with statistical significance after adjustment for other factors. No association was not found in newly diagnosed PDN.

\section{Patterns of Pharmacological Treatment}

In the cross-sectional investigation of pharmacological treatment, $19.52 \%(302 / 1547)$ of participants received prescriptions for neuropathic pain relief. The frequency of pain relief medicines recorded were: gabapentin, $62.86 \%$ (220/350); pregabalin, $12.57 \%$ (44/350), duloxetine, 9.14\% (32/350); ibuprofen, 3.14\% (11/ $350)$; amitriptyline, $2.57 \%$ (9/350); celecoxib, $1.71 \% \quad(6 / 350)$; diclofenac, $1.14 \% \quad$ (4/350); tramadol, 1.14\% (4/350); oxycodone, 1.14\% (4/ $350)$; aceclofenac, $0.86 \%$ (3/350); loxoprofen, $0.86 \%$ (3/350); carbamazepine, $0.57 \%(2 / 350)$; dezocine, $0.57 \%$ (2/350); oxcarbazepine, $0.57 \%$ (2/350); Chinese traditional medicine patch, $0.57 \%$ (2/350); ibuprofen and codeine phosphate, $0.29 \%$ (1/350); and imrecoxib, 0.29\% (1/ $350)$.

The contradiction of guideline recommendations for first-line medicine for pain relief included nonsteroidal anti-inflammatory drugs NSAIDs, Chinese traditional medicine patch and oxcarbazepine, which accounted for $8.29 \%$ (29/350), $0.57 \%(2 / 350)$ and $0.57 \%(2 / 350)$. The second line pain relief of opium account for $2.29 \%(8 / 350)$, the contradiction of guideline recommendation second-linemedicine for pain relief is dezocine $(0.57 \%, 2 / 350)$.

\section{DISCUSSION}

Mainland China has experienced a rapid increase in diabetes prevalence due to economic growth, urbanisation and significant changes in lifestyle. Inevitably, an increase in the prevalence of long-term complications has followed, which are costly both to the individual and the health system. The International Diabetes Federation has predicted that the number of people with diabetes in China, which was 116.4 million in 2019, will increase to 140.5 million by 2030 and 147.2 million by 2045 [8, 9]. PDN management will be a challenge because of the lack of unified early-phase diagnosis standards, insufficient monitoring of glucose control and pain relief and co-morbidites. To meet this challenge, we recently conducted this survey on the clinical characteristics and treatment of PDN.

We assess our findings as a valid elucidation of PDN clinical characteristics and treatment in Mainland China, using a multilevel sampling method. The mean pain severity VAS score was 4.91 , with moderate and severe pain reported for $73.11 \%$ of cases, which is similar to the findings of other research series. Ji et al. reported that the average pain VAS score $( \pm S D)$ for DPN in Chinese studies is $4.12 \pm 2.07$ [10]. Sadosky et al. showed that a mean pain severity 
score for PDN in US studies was 5.2 (0-10 scale) and that $79.5 \%$ of patients reported moderate or severe pain [11].

We have used DN4, a brief and easy-to-use questionnaire that is a particularly amenable tool in our large population of PDN patients, for dissecting neuropathic pain symptoms in epidemiological studies reporting self-reported pain descriptors [12-14]. These items reflect core features of PDN. We found that the symptom of numbness accounted for $81 \%$ of pain phenotype in our study population, which is an indication of neural impairment due to pathological changes. Burning pain, often regarded as a pathognomonic symptom of neuropathic pain, was described by $53.59 \%$ as a pain phenotype; this pain indicates small fibre lesions, degenerative changes in nerve roots or increased excitability through regeneration. The distribution of pain symptoms found in the present survey is similar to that reported previously. An earlier Chinese urban pain status investigation showed that numbness was a prominent descriptor in $64.0 \%$ of patients with DPN, with prickling, pruritus, painful cold and allodynia reported by $24.6,3.4,9.5$ and $8.3 \%$ of patients as pain phenotype, respectively [10]. A French cross-sectional study indicated that the most frequent DN4 sensory phenotypes were numbness, tingling, pins and needles, burning, electric shocks, painful cold and itching [15]. An Italian DPN study reported that the most frequent DN4 sensory phenotypes were tingling, burning, pins and needles, electric shock, painful cold, itching and pain evoked by brushing [16]. The American Standardised Evaluation of Pain phenotypic diversity description [17] implies that sharp, stinging and pins and needles are the most frequent pain descriptions, followed by shooting, throbbing, burning, tenderness, cramping, electric shock, tightness, itching, coldness, spreading, dullness, squeezing, warmth and pulling. Another American study reported the sensory symptoms of numbness and tingling, pricking or pins and needles, shooting pain or sharp jabbing, burning or feeling of heat, stinging or throbbing, electric shock-like feeling and extreme sensitivity to even light touches as the main pain phenotypes [18]. The DN4 phenotype survey used by SCOPE indicated that tingling (68.71\%) and pins and needles $(62.90 \%)$ are prominent symptoms. These symptoms are similar to those reported by French and Italian researchers according to percentage weight, while sharp is more prominent in the USA. In Taiwan, research on sensory descriptors in PDN indicated that the feelings of greatest discomfort were prickling, stabbing, tingling and numbness (a combined symptom percentage) [19]. Dry and painful, burning pain, inexplicable dullness, excruciating pain and 'my leg does not belong to me' were the greatest discomforts felt during sleeping. These were worse at night, any time (or all day long), during walking, when waking up in the morning and during cold days, respectively [19], which is slightly different to that found in the SCOPE survey, especially the majority of reports on prickling, stabbing sensory phenotype. To our knowledge, sociocultural and ethnic differences influence pain perception and responses [20], leading to miscommunication and/or misperception about the presence and/or severity of pain. We hypothesise that majority of differences in sensory phenotype distribution between SCOPE of Mainland China research and research from Taiwan can be attributed to differences in symptom description standardisation or clustering, as well as differences in social culture and dialect.

Opioid use is widely adopted and well managed for the relief of pain in PDN in Mainland China. In our survey, only participants with moderate and severe pain received opium therapies, and the utilisation rate, $0.71 \%(8 / 1131)$, favours avoidance of opioid abuse due to complications, such as opioid overdose, diversion and the development of opioid use disorder. Of the 1547 participants in SCOPE, $19.52 \%(n=$ 302) received pain relief therapy; contradiction of first-line guideline pain relief accounted for $9.43 \%(33 / 350)$ of patients and opium dosage for $0.57 \%(2 / 350)$. These scenarios are similar to those found in other research series. A Korean research into PDN showed that the main pain treatments for PDN were TCA, anticonvulsants, selective serotonin reuptake inhibitors, topical preparations, opioids and serotonin-norepinephrine reuptake inhibitors [21]. A French 
study reported that only $38.6 \%$ of diabetic patients with pain had received appropriate treatment for neuropathic pain [15]. A Belgian study implied that $28 \%$ of patients received appropriate treatment for their neuropathic pain [22]. PDN research in the Czech Republic reported that of 158 patients with PDN, 70 patients were treated with standard analgesics recommended in PDN (44.30\%), whereas 88 patients received either non-recommended analgesia or no treatment [23]. A US healthcare investigation of 3449 PDN cases showed that any pain-related medication for PDN accounted for $91.4 \%$ of cases, opioids for $82.7 \%$, anticonvulsants for $48.2 \%$, NSAIDs for $38 \%$ and antidepressants for 20.9\% during 2012-2013 [24]. A retrospective chart review and crosssectional survey of adult PDN cases in the USA showed that $81.3 \%$ of patients were prescribed at least one medication for their PDN and that $50.9 \%$ reported taking at least one non-prescription medication [11]. The most frequently reported medications for PDN pain relief were anti-epileptics, weak strong-acting opioids (SAO), NSAIDs, strong SAO, long-acting opioids, tramadol, muscle relaxants, serotonin-norepinephrine reuptake inhibitors, topical agents, TCAs and selective serotonin reuptake inhibitors [11]. Regional DPN screening in China, using neurological professional examinations, such as electromyogram, vibration perception, pain, pressure sensation and ankle reflex examination, showed that $32 \%$ of patients with known diabetes mellitus with DPN did not formerly receive any treatment [25]. These SCOPE findings, when compared to the findings of other surveys, suggest that the current pain relief ratio is lower in Mainland China than in other countries. However, contradiction of guideline recommendations for opium prescriptions is much lower in Mainland China than in other countries.

Only a few studies to date have focused on risk factor correlation with VAS pain severity as the dependent variable. The risk factors for the VAS pain severity score in the SCOPE survey were similar to those reported in other studies [26]. Age, female gender, diabetic duration and DN4 score are well-established risk factors for PDN. In our study, PHQ-9 score, depression disorder and GAD-7 score were independently associated with VAS scores among PDN cases, which is identical with previous research [27]. AlQuliti et al. [28] reported age $>50$ years is a risk factor of PDN (odds ratio [OR] 1.93, 95\% confidence interval [CI] 1.09-3.41). Jambart et al. [29] carried out a cross-sectional study and also reported age as a risk factor, with an OR of age 50-64 years of 1.75 (95\%CI 1.48-2.08) and an OR of age $\geq 65$ years of 2.13 (95\% CI 1.72-2.62). However, $\mathrm{Wu}$ et al. [30] did not found age to be an influential factor. Jambart et al. [29] expounded that female gender was a risk factor for PDN (OR 1.27, 95\% CI 1.11-1.46), and Abbott et al. [31] reported the similar result for female gender (OR 1.5, 95\% CI 1.4-1.6). AlQuliti et al. [28] reported diabetic duration was a risk factor for PDN (OR 3.38, 95\% CI 1.88-6.07); Jambart and Van Acker et al. [32] also described diabetic duration as a risk factor for PDN (OR 2.43, 95\% CI 2.10-2.81, OR 1.14, 95\% CI 1.02-1.28). Van Acker [32] reported triglycerides (> $1.7 \mathrm{mmol} / \mathrm{L})(\mathrm{OR} 1.76,95 \% \mathrm{CI}$ 1.13-2.75) and high-density lipoprotein-cholesterol ( $\leq 1 \mathrm{mmol} / \mathrm{L}$ for men, $\leq 1.3 \mathrm{mmol} / \mathrm{L}$ for women) (OR 2.17, 95\% CI 1.38-3.41) to be risk factors for PDN. In our study, we found that hyperlipidemia was a risk factor for PDN; however, we did not define the type. D'Amato et al. [33] conducted a cross-sectional study and reported that depression is a risk factor for PDN (OR 4.56, 95\% CI 1.09-19.1). Gore et al. [34] reported that anxiety (Hospital Anxiety and Depression Scale, HADS) and depression (HADS) are risk factors, which is in agreement with our results although we used the PHQ-9 and GAD-7 scores. Ziegler et al. $[35,36]$ reported that PAD is a risk factor for PDN (OR 9.27, 95\% CI 3.44-25.0, OR 5.61, 95\% CI 2.43-12.96). SCOPE research indicated that $\mathrm{PAD}$ is protective factor for the VAS pain score. The difference in statistical methods used in the Ziegler et al. study and the SCOPE study was that the former defined the presence or absence of neuropathic pain as a dependent variable and the latter used the VAS score; it should be noted that PAD as a predictive factor for VAS score has been seldom reported.

Fasting plasma glucose was demonstrated to be an independent factor associated with PDN 
in a previous study [21]. Some studies have indicated that poor glycaemic control was not associated with PDN [26, 37], whereas other studies found that $\mathrm{HbA} 1 \mathrm{C}$ and pain severity were associated [14]. An interesting discovery from the SCOPE research is that differences in blood glucose level parameters impact on pain severity between newly diagnosed PDN and participants with a previous diagnosis. Veves et al. reported that the natural course of PDN was variable and characterized by neuropathic pain, spontaneous improvement and resolution [38]. Daousi et al. reported that $76 \%$ of patients with PDN still had painful symptoms after 5 years of follow-up [39]. Our results indicate that a previous diagnosis of PDN duration was positively correlated with the VAS pain score and could be attributed to irreversible nerve damage and inadequate pain relief treatment, despite no statistical significance after adjustment for other factors. Diabetic duration was positively correlated with VAS score for all PDN cases, newly diagnosed PDN cases and previously diagnosed PDN, with a statistical significance after adjustment for other factors; this could be attributed to the lack of mechanism therapies and inadequate pain relief treatment. The FBG of previously diagnosed PDN was positively associated with the VAS pain score, but the association was not found in newly diagnosed PDN, indicating that worse FBG blood glucose control is associated with severe pain. We ascribe this to blood glucose control measures for newly diagnosed PDN not being consistently administered.

The present study is the first nationwide, hospital-based study for PDN in Mainland China from the point of view of the neurologist. The unique etiology of DPN includes changes in the expression and distribution of the sodium channel and calcium channels and neuropeptides, sympathetic collateral sprouting, small fiber lesion, microvascular blood flow, abnormalities within the descending pain modulatory periaqueductal gray and rostroventromedial medulla, thalamic neuronal function and neurotransmitters, anterior insula and anterior cingulate cortex, somatosensory cortex and other higher brain centers [40].
To our knowledge, most published studies have focused on diabetic complications, including neuropathy prevalence, and, more recently, on pain status. A nationwide retrospective analysis on chronic diabetic complications and related macrovascular diseases of inpatients with diabetes during 1991-2000 [41] indicated that the prevalence of diabetic sensory neuropathy (including pain, numbness, hypersensitivity, hyposensitivity, single nerve paralysis and palsy) was $60.3 \%$. The reported symptoms included pain, numbness, hypersensitivity, hyposensitivity, single nerve paralysis and palsy. The difference between this retrospective research and SCOPE is the enrolled standard according to the symptoms. Inclusion in SCOPE was based on the neuropathic pain diagnostic criterion [42], with the pain associated with sensory signs in the same neuroanatomically plausible distribution as diagnostic justification. There are several other etiological peripheral neuropathy diseases perhaps confused and differentiated that should be excluded, while the diagnostic justification is according to each sites' medical resources. For example, patients with PAD suffered from rest pain and angina cruris without nerve innervation characteristics; an experienced neurologist could differentiate the disease from PDN by ultrasound artery examination, neural conduction velocity and amplitude.

A pain status study of 565 Chinese urban participants with $>10$ years diabetic duration showed that the morbidity rate of patients with DPN was $46.6 \%$ [10]. The difference between this study and SCOPE and the study is the regional quality of the latter and its standard of diabetic duration. The SCOPE study enrolled outpatients from hospitals, with where the diagnostic practice is different.

There were several limitations to this study. First, the cross-sectional design did not permit exploration of causal inferences between variables and PDN; all results should therefore be considered inferential. Second, the diagnosis of PDN was not assessed with more reliable and quantitative methods, such as nerve conduction studies; rather, the results were derived from possible or probable diagnosis of PDN, and were not confirmed definite diagnose cases [42]. 
Third, although we excluded at enrolment patients with known medical illness causing non-neuropathic or mixed pain, patients may have been included with pain attributable to a different aetiology, as well as non-neuropathic or mixed pain, due to the limited medical resources of outpatient departments.

\section{CONCLUSIONS}

Overall, this SCOPE research not only provides clinically distinctive and characteristic information for PDN in Mainland China but also highlights opportunities for improving management strategies. The study's strength is its external validity, using electronic data from multiple centres, guaranteed by multilevel sampling. The risk factors for $\mathrm{PDN}$, sensory phenotyping and management realities in Mainland China are comparable and although slightly different from those reported in other studies. Numbness, tingling, pins and needles are prominent symptoms in our study population and are slightly different from those reported in other research. PAD as a protective factor for VAS pain score is seldom reported. FBG was found not to be correlated with pain severity of newly diagnosed PDN. Contradiction of guideline recommendations for first-line and second-line pain relief accounted for $9.43 \%$ and $0.57 \%$ of cases, respectively. Opium use is well administrated. The SCOPE results provide a basis for future prospective studies investigating the management of PDN. Identification of modifiable factors of PDN is necessary for the development of early and effective prevention and to halt or reverse its progression.

\section{ACKNOWLEDGEMENTS}

We thank the participants of the study.

Funding. This study process was sponsored by Jiangsu Nhwa Pharmaceutical Co., Ltd. No funding or sponsorship was received for this study or publication of this article. The Rapid Service Fee was funded by the authors.
Authorship. All named authors meet the International Committee of Medical Journal Editors (ICMJE) criteria for authorship for this article, take responsibility for the integrity of the work as a whole, and have given their approval for this version to be published.

Authorship Contributions. We fully acknowledge the participating investigators and active members of the SCOPE Study Group. Dongsheng Fan, Yuanjin Zhang and Nan Li contributed to the concept and design of the research, collected data and contributed to discussions and drafting of the manuscript. Nan Li contributed to the statistics and discussions and reviewed the results. All study participants' data were clinically assessed by Dongsheng Fan, Yuanjin Zhang and Nan Li. Yuanjin Zhang is the guarantor of this work and, as such, had full access to all data in the study and takes responsibility for the integrity of the data and the accuracy of the data analysis. Yuanjin Zhang and other authors are investigators. We especially thank Min Shao, Zheng Zeng, Chunling Su, Xingxia Yang, Fei Teng, Jiao Jing, Pan Xia, Yuzhu Li, Hang Jin, Xiaoli Du, Ting Ding and Yue Hu (Jiangsu Nhwa Pharmaceutical Co., Ltd.) for assistance in the study process. Yingshuang Zhang, Aping Sun (Department of Neurology, Peking University Third Hospital), Yanyin Zhao (Department of Neurology, Huashan Hospital Fudan University) screened participants as investigators.

Disclosures. All authors (Yuanjin Zhang, Shaowei Zhang, Liya Pan, Baojun Wang, Yuanlin Sun, Lijun Gao, Ling Wang, Lijuan Cui, Qing Zhang, Heng Shang, Suqin Jin, Xing Qin, Deqin Geng, Xiaorong Yu, Lin Yang, Li Li, Zuoxiao Li, Chaoli Yan, Hongbin Sun, Tao Sun, Baoxin Du, Junying Cao, Fengyun Hu, Jianhua Ma, Shengnian Zhou, Fengli Zhao, Wei Li, Jianming Zheng, Yanhui Yi, Jianguo Xu, Bo Hu, Baoying Sheng, Zhaohui Li, Zhong Zhao, Ting Yang, Ni Wang, Hongdong Zhao, Dunzhu Mima, Huaiqian Qu, Yi Wang, Fuxia Song, Xinyi Li, Nan Li, Dongsheng Fan) confirm that they have no conflicts of interest to disclose. 
Compliance with Ethics Guidelines. All participants volunteered to participate in the SCOPE study and gave written, informed consent. Master ethical approval was obtained from the Peking University Third Hospital Medical Science Research Ethics Committee (Record Number: IRB00006761-M2018029, approval number: 2018-182-01). The study was performed in accordance with the Helsinki Declaration of 1964 and its later amendments. The ClinicalTrials.gov registration number is NCT03520608 (2018-05-11). Participating hospitals also acquired local site ethical approval. Ethic committees of several participating centres adopted a registry system without a reference number. Several ethics committees accepted the common practice that retrospective research did not require ethics approval. One survey participant was not approved for continuing investigation, and the data on this patient were not included in the statistical analysis.

Data Availability. The datasets generated during and/or analyzed during the current study are available from the corresponding author on reasonable request.

Open Access. This article is licensed under a Creative Commons Attribution-NonCommercial 4.0 International License, which permits any non-commercial use, sharing, adaptation, distribution and reproduction in any medium or format, as long as you give appropriate credit to the original author(s) and the source, provide a link to the Creative Commons licence, and indicate if changes were made. The images or other third party material in this article are included in the article's Creative Commons licence, unless indicated otherwise in a credit line to the material. If material is not included in the article's Creative Commons licence and your intended use is not permitted by statutory regulation or exceeds the permitted use, you will need to obtain permission directly from the copyright holder. To view a copy of this licence, visit http://creativecommons.org/licenses/by$\mathrm{nc} / 4.0 /$.

\section{REFERENCES}

1. Boulton AJ, Vileikyte L, Ragnarson-Tennvall G, Apelqvist J. The global burden of diabetic foot disease. Lancet. 2005;366:1719-24.

2. Izenberg A, Perkins BA, Bril V. Diabetic neuropathies. Semin Neurol. 2015;35(4):424-30.

3. Zhang Y, Li N, Zhao Y, et al. Painful Diabetic Peripheral Neuropathy Study of Chinese OutPatiEnts (PDN-SCOPE): protocol for a multicentre cross-sectional registry study of clinical characteristics and treatment in China. BMJ Open. 2019;9: e025722.

4. Wang YF, Yang CC, Ro LS, et al. Development and validation of a Taiwan version of the DN4-T questionnaire. J Chin Med Assoc. 2019;82(8):623-7.

5. Tesfaye S, Vileikyte L, Rayman G, et al. Painful diabetic peripheral neuropathy: consensus recommendations on diagnosis, assessment and management. Diabetes Metab Res Rev. 2011;27:629-38.

6. Bril V, England J, Franklin GM, et al. Evidencebased guideline: treatment of painful diabetic neuropathy report of the American Academy of Neurology, the American Association of Neuromuscular and Electrodiagnostic Medicine, and the American Academy of Physical Medicine and Rehabilitation Neurology. PM R. 2011;76:1-1.

7. Handelsman Y, Bloomgarden ZT, Grunberger G, et al. American Association of Clinical Endocrinologists and American College of Endocrinologyclinical practice guidelines for developing a diabetes mellitus comprehensive care plan-2015. Endocr Pract. 2015;21(Suppl 1):1-87.

8. Shaw JE, Sicree RA, Zimmet PZ. Global estimates of the prevalence of diabetes for 2010 and 2030. Diabetes Res Clin Pract. 2010;87(1):4-14.

9. Saeedi P, Petersohn I, Salpea P, et al. Global and regional diabetes prevalence estimates for 2019 and projections for 2030 and 2045: Results from the International Diabetes Federation Diabetes Atlas, 9th edition. Clin Pract. 2019;157:107843.

10. Na J, Nan Z, Zhan RJ, et al. Risk factors and pain status due to diabetic neuropathy in chronic longterm diabetic patients in a Chinese urban population. Chin Med J. 2012;125(23):4190-6.

11. Sadosky A, Schaefer C, Mann R, et al. Burden of illness associated with painful diabetic peripheral neuropathy among adults seeking treatment in the US: results from a retrospective chart review and cross-sectional survey. Diabetes Metab Syndr Obes Targets Ther. 2013;6:79-92. 
12. Gylfadottir SS, Christensen DH, Nicolaisen SK, et al. Diabetic polyneuropathy and pain, prevalence, and patient characteristics: a cross-sectional questionnaire study of 5514 patients with recently diagnosed type 2 diabetes. Pain. 2019;161:1-10.

13. Spallone V, Morganti R, D'Amato C, et al. Validation of DN4 as a screening tool for neuropathic pain in painful diabetic polyneuropathy. Diabet Med. 2012;29:578-85.

14. Themistocleous AC, Ramirez JD, Shillo PR, et al.. The Pain in Neuropathy Study (PiNS): a crosssectional observational study determining the somatosensory phenotype of painful and painless diabetic neuropathy. Pain. 2016;157:1132-45.

15. Bouhassira D, Letanoux M, Hartemann A. Chronic pain with neuropathic characteristics in diabetic patients: a French cross-sectional study. PLoS ONE. 2013;8(9):e74195.

16. Truini A, Spallone V, Morganti R, et al. A crosssectional study investigating frequency and features of definitely diagnosed diabetic painful polyneuropathy. Pain. 2018;159:2658-66.

17. Scholz J, Rathmell JP, David WS, et al. A standardized clinical evaluation of phenotypic diversity in diabetic polyneuropathy. Pain. 2016;157:2297-308.

18. Sadosky A, Hopper J, Parsons B. Painful diabetic peripheral neuropathy: results of a survey characterizing the perspectives and misperceptions of patients and healthcare practitioners. Patient. 2014;7:107-14.

19. Jane S-W, Lin M-S, Chiu W-N, et al. Prevalence, discomfort and self-relief behaviours of painful diabetic neuropathy in Taiwan: a cross-sectional study. BMJ Open. 2016;6:e011897.

20. Shavers VL, Bakos A, Sheppard VB. Race, ethnicity, and pain among the US adult population. J Health Care Poor Underserved. 2010;21:177-220.

21. Kim SS, Won JC, Kwon HS, et al. Prevalence and clinical implications of painful diabetic peripheral neuropathy in type 2 diabetes: results from a nationwide hospital-based study of diabetic neuropathy in Korea. Diabetes Res Clin Pract. 2014;103(3):522-9.

22. Kaur S, Pandhi P, Dutta P, et al. Painful diabetic neuropathy: an update. Ann Neurosci. 2011;18: 168-75.

23. Raputova J, Srotova I, Vlckova E, et al. Sensory phenotype and risk factors for painful diabetic neuropathy: a cross-sectional observational study. Pain. 2017;158:2340-53.
24. Raputova J, Srotova I, Vlckova E, et al. Sensory phenotype and risk factors for painful diabetic neuropathy: a cross-sectional observational study. Pain. 2017;158(12):2340-53.

25. Sadosky A, Mardekian J, Parsons B, et al. Healthcare utilization and costs in diabetes relative to the clinical spectrum of painful diabetic peripheral neuropathy. J Diabetes Complications 2015;29: 212-7.

26. Hebert HL, Veluchamy A, Torrance N, et al. Risk factors for neuropathic pain in diabetes mellitus. Pain. 2017;158:560-8.

27. Jain R, Jain S, Raison CL, Maletic V. Painful diabetic neuropathy is more than pain alone: examining the role of anxiety and depression as mediators and complicators. Curr Diabet Rep. 2011;11(4):275-84.

28. AlQuliti K. Predictors of painful diabetic neuropathy in Saudi patients with type 2 diabetes. J Pain Relief. 2015;4:181.

29. Jambart S, Ammache Z, Haddad F, et al. Prevalence of painful diabetic peripheral neuropathy among patients with diabetes mellitus in the Middle East region. J Int Med Res. 2011;39:366-77.

30. Wu EQ, Borton J, Said G, et al. Estimated prevalence of peripheral neuropathy and associated pain in adults with diabetes in France. Curr Med Res Opin. 2007;23:2035-42.

31. Abbott CA, Malik RA, van Ross ER, Kulkarni J, Boulton AJ. Prevalence and characteristics of painful diabetic neuropathy in a large community based diabetic population in the UK. Diabet Care. 2011;34:2220-4.

32. Van Acker K, Bouhassira D, De Bacquer D, et al. Prevalence and impact on quality of life of peripheral neuropathy with or without neuropathic pain in type 1 and type 2 diabetic patients attending hospital outpatients clinics. Diabet Metab. 2009;35: 206-13.

33. D'Amato C, Morganti R, Greco C, et al. Diabetic peripheral neuropathic pain is a stronger predictor of depression than other diabetic complications and comorbidities. Diabet Vasc Dis Res. 2016;13: 418-28.

34. Gore M, Brandenburg NA, Dukes E, Hoffman DL, Tai KS, Stacey B. Pain severity in diabetic peripheral neuropathy is associated with patient functioning, symptom levels of anxiety and depression, and sleep. J Pain Symptom Manag. 2005;30:374-85.

35. Ziegler D, Rathmann W, Dickhaus T, Meisinger C, Mielck A. Neuropathic pain in diabetes, prediabetes and normal glucose tolerance: the MONICA/KORA 
Augsburg Surveys S2 and S3. Pain Med. 2009;10: 393-400.

36. Ziegler D, Rathmann W, Meisinger C, Dickhaus T, Mielck A. Prevalence and risk factors of neuropathic pain in survivors of myocardial infarction with prediabetes and diabetes. The KORA Myocardial Infarction Registry. Eur J Pain. 2009;13:582-7.

37. Ponirakis G, Elhadd T, Chinnaiyan S, et al. Prevalence and risk factors for diabetic neuropathy and painful diabetic neuropathy in primary and secondary healthcare in Qatar. J Diabetes Investig. 2021;12(4):592-600.

38. Veves A, Backonja M, Malik RA. Painful diabetic neuropathy: epidemiology, natural history, early diagnosis, and treatment options. Pain Med. 2008;9(6):660-74.
39. Daousi C, Benbow SJ, Woodward A, MacFarlane IA. The natural history of chronic painful peripheral neuropathy in a community diabetes population. Diabet Med. 2006;23(9):1021-4.

40. Shillo P, Sloan G, Greig M, et al. Painful and painless diabetic neuropathies: what is the difference? Curr Diabet Rep. 2019;19(6):32.

41. Weng JP, Bi Y. Epidemiological status of chronic diabetic complications in China. Chin Med J. 2015;128:3267-9.

42. Finnerup NB, Haroutounian S, Kamerman P, et al. Neuropathic pain: an updated grading system for research and clinical practice. Pain. 2016;157(8): 1599-606. 